\title{
Smoking duration alone provides stronger risk estimates of chronic obstructive pulmonary disease than pack-years
}

\author{
Surya P Bhatt, ${ }^{1,2}$ Young-il Kim, ${ }^{3}$ Kathy F Harrington, ${ }^{1}$ John E Hokanson, ${ }^{4}$ \\ Sharon M Lutz, ${ }^{4}$ Michael H Cho, ${ }^{5}$ Dawn L DeMeo, ${ }^{5,6,7}$ James M Wells, ${ }^{1,2}$ Barry J Make, ${ }^{8}$ \\ Stephen I Rennard, ${ }^{9,10}$ George R Washko, ${ }^{6,7}$ Marilyn G Foreman, ${ }^{11}$ Donald P Tashkin, ${ }^{12}$ \\ Robert A Wise, ${ }^{13}$ Mark T Dransfield, ${ }^{1,2,14}$ William C Bailey, ${ }^{1,2}$ On behalf of the \\ COPDGene Investigators
}

\begin{abstract}
- Additional material is published online only. To view please visit the journal online (http://dx.doi.org/10.1136/ thoraxjn-2017-210722)

For numbered affiliations see end of article.
\end{abstract}

Correspondence to Dr Surya P Bhatt, Division of Pulmonary, Allergy and Critical Care Medicine, University of Alabama at Birmingham, Birmingham, AL 35294, USA; sbhatt@uabmc.edu

Received 3 July 2017 Revised 3 November 2017 Accepted 27 November 2017 Published Online First 11 January 2018

\section{Linked}

http://dx.doi.org/10.1136/ thoraxjnl-2017-211356

Check for updates

To cite: Bhatt SP, Kim Y, Harrington KF, et al. Thorax 2018:73:414-421

\section{ABSTRACT}

Background Cigarette smoking is the strongest risk factor for COPD. Smoking burden is frequently measured in pack-years, but the relative contribution of cigarettes smoked per day versus duration towards the development of structural lung disease, airflow obstruction and functional outcomes is not known. Methods We analysed cross-sectional data from a large multicentre cohort (COPDGene) of current and former smokers. Primary outcome was airflow obstruction (FEV /FVC); secondary outcomes included five additional measures of disease: $\mathrm{FEV}_{1}, \mathrm{CT}$ emphysema, CT gas trapping, functional capacity (6 min walk distance, 6MWD) and respiratory morbidity (St George's Respiratory Questionnaire, SGRQ). Generalised linear models were estimated to compare the relative contribution of each smoking variable with the outcomes, after adjustment for age, race, sex, body mass index, CT scanner, centre, age of smoking onset and current smoking status. We also estimated adjusted means of each outcome by categories of pack-years and combined groups of categorised smoking duration and cigarettes/ day, and estimated linear trends of adjusted means for each outcome by categorised cigarettes/day, smoking duration and pack-years.

Results 10187 subjects were included. For FEV,/FVC, standardised beta coefficient for smoking duration was greater than for cigarettes/day and pack-years $(P<0.001)$. After categorisation, there was a linear increase in adjusted means $F E V$, $/ F V C$ with increase in pack-years (regression coefficient $\beta=-0.023 \pm S E 0.003 ; P=0.003$ ) and duration over all ranges of smoking cigarettes/day $(\beta=-0.041 \pm 0.004 ; P<0.001)$ but a relatively flat slope for cigarettes/day across all ranges of smoking duration $(\beta=-0.009 \pm 0.0 .009 ; P=0.34)$. Strength of association of duration was similarly greater than pack-years for emphysema, gas trapping, FEV, $6 \mathrm{MWD}$ and SGRQ.

Conclusion Smoking duration alone provides stronger risk estimates of COPD than the composite index of pack-years. Trial registration number Post-results; NCT00608764.

\section{BACKGROUND}

COPD is characterised by partially reversible airflow obstruction and is usually associated with

\section{Key messages}

What is the key question?

- What is the relative contribution of cigarettes smoked per day versus duration towards the development of COPD?

What is the bottom line?

- In a population of current and former smokers, we found that although the average number of cigarettes smoked per day is important, smoking duration alone is more strongly associated with COPD disease components than pack-years, and that cigarettes/day may lower the strength of association between cigarette smoking and COPD metrics in epidemiological studies. Our findings have significant public health implications and might make smoking history ascertainment easier as well as more likely to be accurate.

Why read on?

- We show that due to difficulties in ascertainment of cigarettes/day, smoking duration is more strongly associated with COPD than the composite of pack-years.

structural changes on lung imaging. ${ }^{1}$ Although the demonstration of airflow obstruction on spirometry is sine qua non for the diagnosis of COPD, the pretest probability of a correct diagnosis relies on accurate quantification of risk factors as well as symptom burden. Cigarette smoking is the strongest risk factor for COPD, ${ }^{1}$ and although no precise estimate of a threshold effect is available, in those smokers who develop COPD there is a dose-effect relationship. Various parameters of tobacco exposure have been proposed including urinary and salivary cotinine estimations; however, these are expensive and do not provide accurate estimates of the cumulative exposure. ${ }^{2}$ COPD is a disease with a long latency period and hence questionnaires are best suited for assessing exposure over time. ${ }^{3}$

Smoking burden is frequently measured in packyears, a product of the average number of packs 
of cigarettes smoked a day and smoking duration in years. The exposure time risk profile for some diseases with a clear onset of disease has been delineated. For example, lung cancer is associated more strongly with the duration of smoking than with the average cigarettes/day. ${ }^{4-6}$ This delineation provides mechanistic insights and has implications for tobacco cessation strategies. The pack-years product assumes equal weightage for both cigarettes/day and duration of smoking, and the relative contribution of cigarettes/day versus duration towards the development of COPD is not known. Although ideally this is best answered by studying onset of smoking to onset of disease, COPD remains undiagnosed in the majority of patients long after the onset of disease and hence we adapted the design of comparing disease severity across a range of smoking burden. We hypothesised that cigarettes smoked per day and smoking duration differentially impact the occurrence and severity of COPD disease components including airflow obstruction, structural lung disease and functional outcomes.

\section{METHODS}

\section{Study population}

We analysed cross-sectional data from a large multicentre cohort (COPDGene), the details of this study have been previously published. ${ }^{7}$ Briefly, participants in COPDGene were of self-identified non-Hispanic White or African-American racial/ ethnic category, and included current and former smokers (ages 45-80 years) with at least 10 pack-year smoking history. Participants with other known lung diseases other than asthma were excluded; for example, pulmonary fibrosis, extensive bronchiectasis, and cystic fibrosis, previous surgical excision of at least one lung lobe (or lung volume reduction procedure), active cancer under treatment, suspected lung cancer (large or highly suspicious lung mass), metal in the chest, recent exacerbation of COPD treated with antibiotics or steroids, recent eye surgery, recent myocardial infarction or other cardiac hospitalisation, recent chest or abdominal surgery, inability to use albuterol, history of chest radiation therapy, and first or second-degree relative already enrolled in the study. Written informed consent was obtained from all participants prior to enrolment in the cohort. Those with known lung disease except for COPD and asthma were excluded. Detailed assessments of smoking history were recorded as reported by the participants at the time of enrolment including age at which participants started smoking, duration of smoking (calculated as the difference between the age at time of quitting or time of enrolment and the age at smoking initiation) and the average number of cigarettes a day. Smoking burden was also assessed using the traditional metric of pack-years, which is the product of the average number of packs of cigarettes a day and smoking duration in years. To lessen the effect of early-onset smoking on the developing lung, we also separately analysed participants who started smoking at or after the age of 18 years.

\section{Measurement of disease}

The primary outcome was airflow obstruction measured by the ratio of the $\mathrm{FEV}_{1}$ to the FVC (FEV $\left./ \mathrm{FVC}\right)$. Secondary outcomes included five additional measures of disease: $\mathrm{FEV}_{1}$, CT emphysema, CT gas trapping, functional capacity using $6 \mathrm{~min}$ walk distance (6MWD) and respiratory morbidity using St George's Respiratory Questionnaire (SGRQ). Presence of COPD was primarily assessed by spirometric and imaging metrics, and disease burden quantitated using respiratory quality of life and functional capacity measurements. COPD was defined by the Global Initiative for Chronic Obstructive Lung Disease (GOLD) criteria of postbronchodilator
$\mathrm{FEV}_{1} / \mathrm{FVC}<0.70$. Participants with normal ratio but $\mathrm{FEV}_{1}<80 \%$ predicted were categorised to have GOLD unclassifiable disease or preserved ratio impaired spirometry. ${ }^{8}$

CT scans were performed at full inspiration (total lung capacity, TLC) and end-tidal exhalation (functional residual capacity, FRC). After segmentation and extrusion of the large and medium-sized airways, emphysema was quantified as the percentage of lung volume at TLC with attenuation <-950 Hounsfield units (HU) by density mask analyses using 3D Slicer software (http://airwayinspector.acil-bwh.org/). ${ }^{7}$ CT measures of emphysema correlate well with emphysema measured on histopathology. ${ }^{9}$ Gas trapping was quantified as the percentage of lung volume at FRC with attenuation $<-856 \mathrm{HU}$. Respiratory-related quality of life was assessed using SGRQ; SGRQ ranges from 0 to 100 with higher scores indicating worse respiratory-related quality of life. ${ }^{10}$ We assessed functional capacity using the 6MWD according to the American Thoracic Society (ATS) guidelines. $^{11}$

\section{Statistical analyses}

We analysed the data using two methods. (See online supplementary information for details.) First, using general linear models, we estimated association of each outcome with the smoking variables (duration, cigarettes/day and pack-years) as continuous variables in separate fixed-effects models after adjustment for age, race, sex, body mass index, CT scanner type, centre, age of smoking onset and current smoking status. ${ }^{12}$ To estimate relative effect sizes, we compared the standardised regression coefficients of the smoking variables for associations with the outcome. $\mathrm{FEV}_{1} / \mathrm{FVC}$ was the primary outcome, and we repeated these analyses for each of the secondary outcomes (CT emphysema, CT gas trapping, FEV, 6MWD and SGRQ). Results were determined to be statistically significant when the accompanying statistical test yielded a probability of $<0.05$.

Second, to improve clinical interpretability and applicability across a range of combination of smoking duration and cigarettes/day, we categorised the smoking variables by increments of 10 units to create consistent intervals for all three of the smoking variables. The six metrics of disease described above were treated as outcome variables and their adjusted means were calculated for each grouping of 23 combinations of smoking duration and cigarettes/day using a $5 \times 5$ table $(25$ groups were designed and two groups are not evaluable due to zero or insufficient cases). Similarly, adjusted means of outcomes were estimated for five groups of smoking pack-years. Covariates used for adjusting means of the outcomes were age, race, sex, body mass index, CT scanner type, centre, age of smoking onset and current smoking status. Linear trends of the adjusted means of outcomes over categorised smoking duration and cigarettes/day were then drawn to illustrate the trends of the 23 adjusted means of the outcomes over cigarettes/day and duration, respectively (online supplementary table 1). These trends provide information on the relative contribution of one smoking variable towards COPD when the other smoking variable is held constant. In addition, linear trends were estimated for the adjusted means of outcomes over five groups of pack-years. Steepness of linear slope over pack-years was graphically illustrated for comparison with linear slopes over duration and cigarettes/day. All analyses were performed using SAS V.9.4 (SAS Institute).

\section{RESULTS}

\section{Participant characteristics}

10300 subjects were enrolled in the COPDGene study. We excluded 108 participants who were non-smokers. A total 


\begin{tabular}{lc}
\hline \multicolumn{1}{l}{ Table 1} & \\
\hline Parameters & $\mathrm{n}=10187$ \\
\hline Age (years) & $59.6(9.0)$ \\
\hline Sex, female (\%) & $4741(46.5)$ \\
\hline Race, African American (\%) & $3407(33.4)$ \\
\hline Body mass index (kg/m²) & $28.8(6.3)$ \\
\hline Smoking pack-years & $44.2(25.0)$ \\
\hline Smoking duration (years) & $36.4(10.0)$ \\
\hline Cigarettes/day & $24.3(11.3)$ \\
\hline Current smokers (\%) & $5414(53.2)$ \\
\hline FEV ${ }_{1}$ (L) & $2.24(0.92)$ \\
\hline FEV ${ }_{1} \%$ predicted & $76.4(25.6)$ \\
\hline FVC (L) & $3.30(1.01)$ \\
\hline FVC \% predicted & $87.0(18.3)$ \\
\hline FEV $/$ /FVC & $0.67(0.16)$ \\
\hline CT emphysema (\%) & $6.2(9.6)$ \\
\hline CT gas trapping (\%) & $21.9(19.9)$ \\
\hline 6 min walk distance (m) & $411.5(121.6)$ \\
\hline St George's Respiratory Questionnaire score (units) & $27.4(22.9)$ \\
\hline Al Valles a expressed & \\
\hline
\end{tabular}

All values are expressed as mean (SD) unless otherwise indicated.

of 10187 subjects were included in the current analyses after excluding five subjects who were placed in the category with smoking duration $0-10$ years and smoking cigarettes/day average of $\geq 40.1$ cigarettes/day, due to insufficient number of cases. Characteristics of participants included are shown in table 1. The mean age at enrolment was 59.6 (9.0) years. 5446 (53.5\%) were male and 3407 (33.4\%) were African American. The cohort included 5414 (53.2\%) current smokers and 4773 (46.8\%) former smokers. The mean pack-years of smoking was 44.2 (25.0) (IQR 27.2-54.9). The average duration of smoking was 36.4 (10.0) years (IQR 30.9-43.0 years), and mean cigarettes/day was 24.3 (11.3) (IQR 20.0-30.0). There were weak correlations between age and pack-years of smoking $(r=0.266)$, smoking duration $(\mathrm{r}=0.342)$ and cigarettes/day $(\mathrm{r}=0.134)$. Online supplementary table 2 shows smoking burden by cigarettes/day and duration across GOLD stages.

\section{Smoking and airflow obstruction}

We found that for the degree of airflow obstruction $\left(\mathrm{FEV}_{1} /\right.$ FVC), the adjusted effect size was greater for smoking duration than for cigarettes/day and pack-years. After categorisation, we found a linear increase in $\mathrm{FEV}_{1} / \mathrm{FVC}$ with increase in pack-years $(\beta=-0.023 \pm$ SE0.003; $P=0.003)$. With increasing cigarettes/day, the gradient remained relatively flat across all ranges of smoking duration $(\beta=-0.009 \pm 0.009 ; \mathrm{P}=0.34)$ (figure $1 \mathrm{~A})$. Smoking duration also had a linear inverse relationship with $\mathrm{FEV}_{1} / \mathrm{FVC}$ $(\beta=-0.041 \pm 0.004 ; P<0.001)$, and its slope was again steeper than pack-years for association with $\mathrm{FEV}_{1} / \mathrm{FVC}$ (figures $1 \mathrm{~B}$ and 2A). Similar results were seen for $\mathrm{FEV}_{1}$ (figure 2B).

\section{Smoking and structural lung disease on CT}

For CT emphysema, the adjusted effect size was greater for smoking duration than for cigarettes/day and pack-years (table 2). The root mean square error (RMSE) for the model with duration was less than RMSE for cigarettes/day and pack-years. On categorisation of the data, there was a linear increase in adjusted means per cent emphysema with increase in pack-years (regression coefficient $\beta=1.219 \pm$ SE0.099; $\mathrm{P}=0.001$ ) (figures 2C and 3). With increasing cigarettes/day, the slope remained relatively flat across all ranges of smoking duration $(\beta=0.377 \pm 0.437 ; \mathrm{P}=0.40)$ (figures $1 \mathrm{C}$ and 3$)$. Smoking duration also had a linear relationship with per cent emphysema $(\beta=1.937 \pm 0.151 ; P<0.001)$, and its slope was steeper than pack-years for association with emphysema (figures $1 \mathrm{C}$ and $2 \mathrm{C}$ ).

Similarly for gas trapping, the effect size was greater for smoking duration than for cigarettes/day and pack-years (table 2). After categorisation, there was a linear increase in the per cent gas trapping with increase in pack-years $(\beta=2.484 \pm$ SE0.291; $P=0.003$ ) (figure 2D). The slope remained comparatively flat across all ranges of smoking duration with increasing cigarettes/day $(\beta=1.001 \pm 0.960 ; P=0.31)$. Smoking duration also had a linear relationship with per cent gas trapping $(\beta=4.254 \pm 0.352 ; P<0.001)$, and its slope was again steeper than pack-years for association with gas trapping (figure 2D).

\section{Smoking and respiratory morbidity}

The effect size was greater for smoking duration than for cigarettes/day and pack-years for association with SGRQ (table 2). SGRQ also linearly worsened with increase in pack-years $(\beta=3.292 \pm$ SE0.418; $P=0.004)$. Unlike the relationship with other disease measures, cigarettes/day was associated with worse respiratory quality of life $(\beta=2.696 \pm 1.111 ; \mathrm{P}=0.024)$. SGRQ linearly worsened with greater smoking duration $(\beta=4.781 \pm 0.720 ; P<0.001)$, and this slope was steeper than pack-years for association with SGRQ (figure $2 \mathrm{E}$ ).

6MWD decreased in a linear fashion with increase in packyears $(\beta=-38.392 \pm$ SE4.500; $P=0.003)$. With increasing cigarettes/day, the slope remained relatively flat across all ranges of smoking duration $(\beta=-22.361 \pm 15.903 ; \mathrm{P}=0.17)$. 6MWD also decreased linearly with smoking duration $(\beta=-67.617 \pm 7.979 ; P<0.001)$, and this slope was steeper than that for pack-years (figure $2 \mathrm{~F}$ ). The effect size was greater for smoking duration than for cigarettes/day and pack-years for association with $6 \mathrm{MWD}$. Figure 2 shows a comparison of slopes for outcomes per each smoking variable.

\section{Secondary analyses}

To examine whether the age of smoking onset impacted the results, we performed sensitivity analyses by repeating these analyses to test the relationship between smoking variables and disease metrics, in participants who started smoking at or after the age of 18 (online supplementary table 3), as well as for the entire cohort without adjustment for age of smoking onset (online supplementary table 4). We found that for both of these analyses, the linear trends for smoking duration were steeper for COPD outcomes than pack-years, and that the slope for cigarettes/day was relatively flat.

\section{DISCUSSION}

In a cohort of current and former smokers, we showed that smoking duration alone is more strongly associated with estimates of COPD disease components than cigarettes smoked per day, and the composite index of pack-years. Although cigarettes/ day is also associated with COPD, using pack-years as measured currently may lower the strength of association between cigarette smoking and COPD metrics in epidemiological studies.

Measuring smoking burden using pack-years has long been recognised to be imprecise. The relative contributions of smoking duration and cigarettes/day have been examined in other smoking-related diseases, but with relatively easily recognised disease 


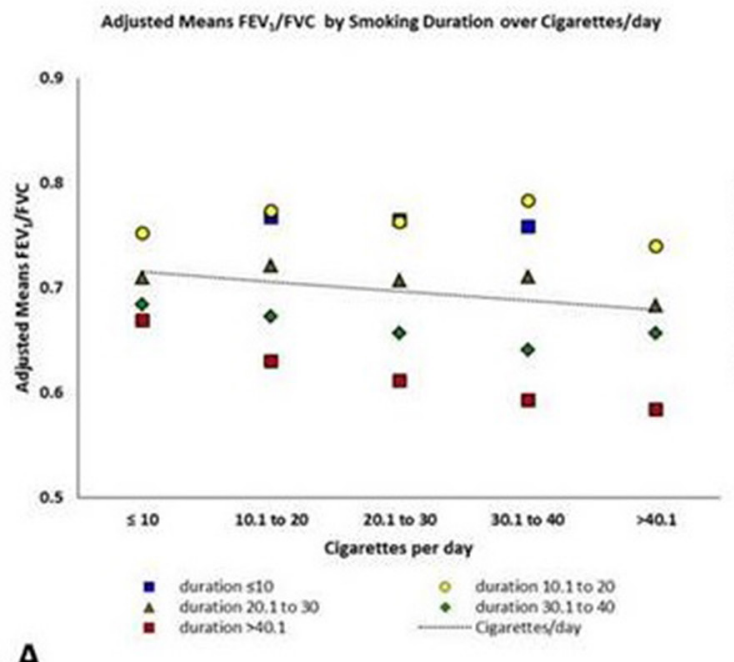

A

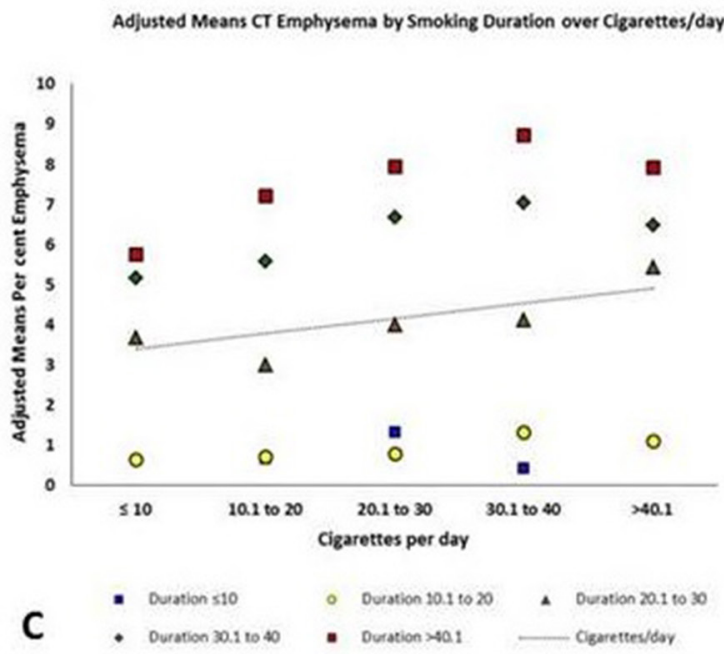

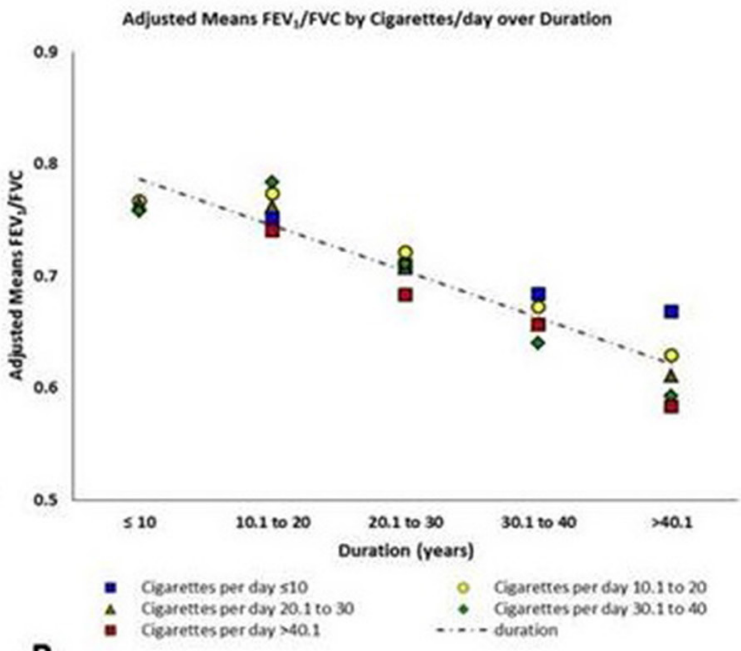

Adjusted Means Cr Emphysema by Cigarettes/day over Duration

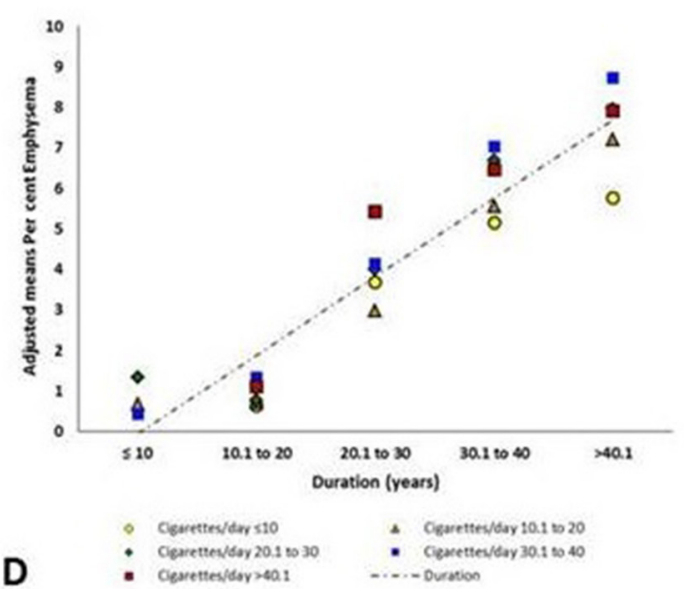

Figure 1 Panel (A) shows linear slopes for adjusted means of $\mathrm{FEV}_{1} / \mathrm{FVC}$ over categorised cigarettes/day. Panel (B) shows linear slopes for adjusted means of $\mathrm{FEV}_{1} / \mathrm{FVC}$ over categorised duration. Colour-coded data points represent estimated adjusted means of FEV/FVC by categorised duration (A) or cigarettes/day (B). All categorisation is based on 10 unit increments. Panel (C) shows linear slopes for adjusted means of CT emphysema over categorised cigarettes/day. Panel (D) shows linear slopes for adjusted means of CT emphysema over categorised smoking duration. Colour-coded data points represent estimated adjusted means of CT emphysema by categorised duration (C) or cigarettes/day (D). All categorisation is based on 10 unit increments. Least square means of FEV,/FVC and CT emphysema are adjusted for age, race, sex, body mass index, scanner type, centre, age of smoking onset and current smoking status.

onset such as lung cancer and oesophageal cancer. ${ }^{4-6} 13$ For both these cancers, smoking duration was more strongly associated with disease than cigarettes/day, but no comparisons were made to assess the strength of these associations with pack-years. Similar results have been reported for cardiovascular disease for which smoking duration appears to be more important than cigarettes/day, without direct comparisons with pack-years. ${ }^{14}$ Results from the Lung Health Study showed that increasing cigarettes/day is associated with greater lung function decline in mild to moderate COPD, but no direct comparisons were made between cigarettes/day and duration of smoking. ${ }^{15} 16$ We found that smoking duration is more important for presence of disease in a chronic slowly evolving disease, and that cigarettes/day as currently recorded may attenuate the strength of association between cigarette smoking and disease measures. The reasons for this finding could be biological or epidemiological. It is plausible that a longer duration of smoking is associated with increasing accumulation of genetic and epigenetic changes, especially in this polygenic condition. ${ }^{17}$ There may also be alterations in the lung microbiome over time with continued cigarette smoke exposure ${ }^{18}$; there is lesser microbial diversity with continued cigarette smoking and this may contribute to disease progression. ${ }^{19}$ Epidemiologically, smoking burden has been estimated in several ways, including prospective direct methods such as personal monitoring in smokers' microenvironments and biochemical assays such as plasma, urinary and salivary cotinine levels. ${ }^{3}$ These are limited by feasibility and costs especially in COPD which has a long latency period, as well as non-availability of measurements of time of exposure. Inherent to the long duration of smoking, smoking history is almost always self-reported and hence subject to recall bias. We believe that the duration of smoking is more easily recalled than the average cigarettes/day of smoking which tends to fluctuate over time. Cigarettes smoked per day is also harder to quantify accurately and cigarettes/day measurements correlate poorly with biochemical assessments of smoking exposure. ${ }^{2}$ This could be due to different formulations of cigarettes 


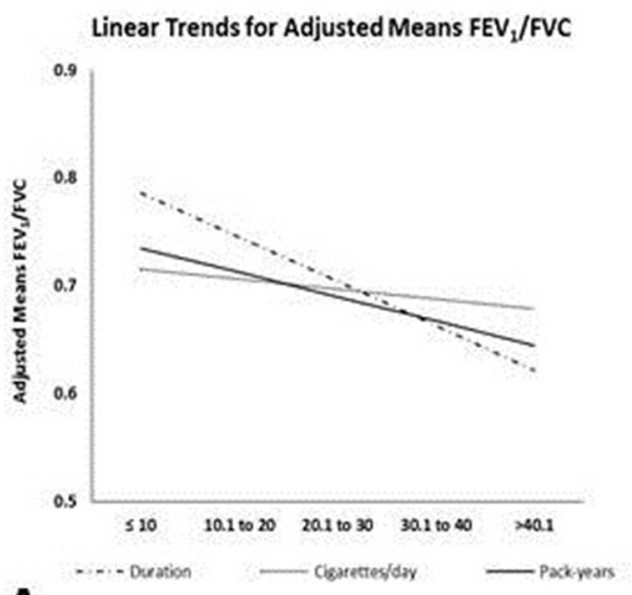

A
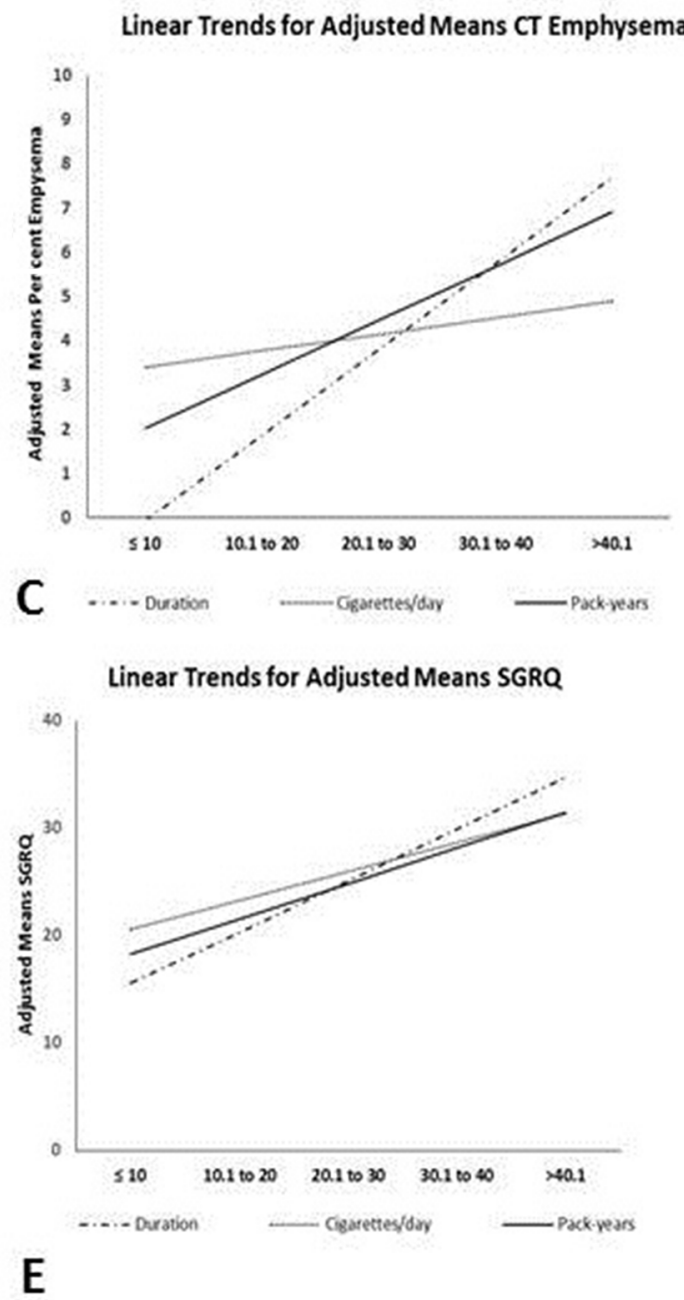
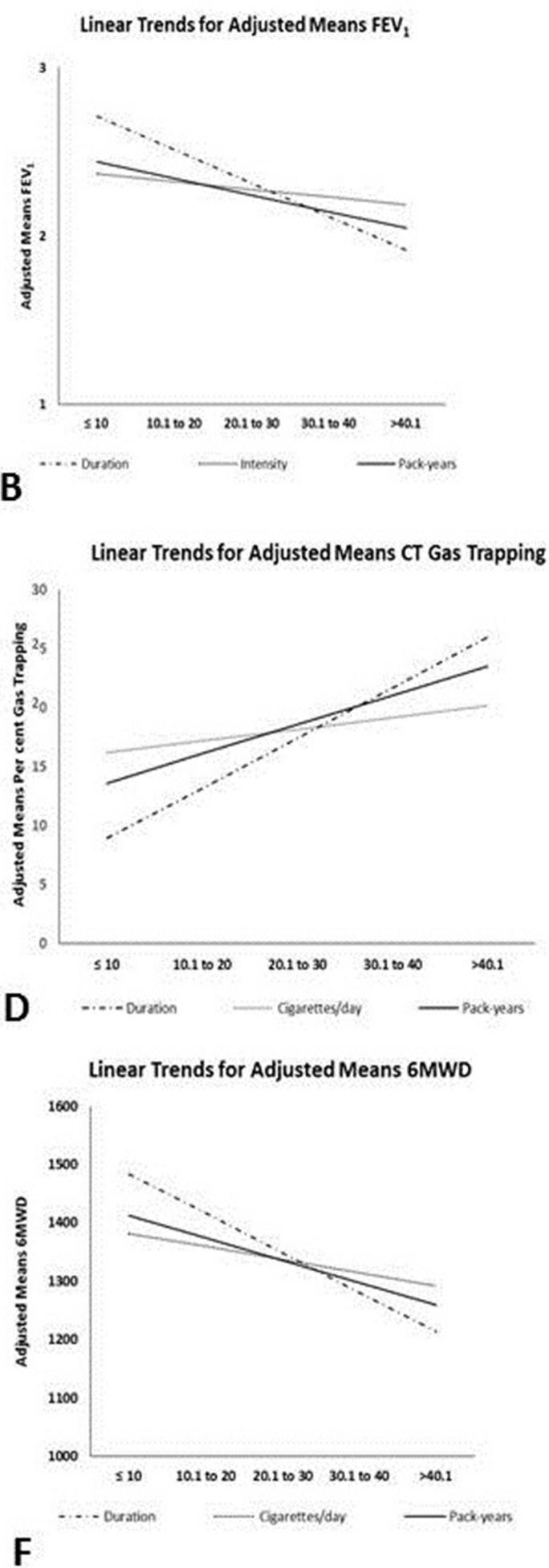

Figure 2 Summary linear slopes for lung function ((A) FEV /FVC and (B) FEV $)_{1}$, structural lung disease ((C) CT emphysema and (D) CT gas trapping), and quality of life ((E) SGRQ) and exercise capacity ((F) 6MWD) over categorised cigarettes/day, categorised smoking duration and over categorised pack-years. All categorisation is based on 10 unit increments. All outcome least square means adjusted for age, race, sex, body mass index, scanner type, centre, age of smoking onset and current smoking status. 6MWD, 6 min walk distance; SGRQ, St George's Respiratory Questionnaire.

and different amounts of exposure that depend on mainstream and sidestream smoke, with the latter likely more dangerous. ${ }^{2}$ Smoking topography is heterogeneous and many smokers adjust the level of smoke inhaled, further affecting measurement. ${ }^{20}$ It is also possible that the 'bandwidth' of cigarettes smoked per day is constrained by increments of number of cigarettes by half to one pack, although we did have participants reporting increments of single units of average cigarettes smoked per day, and hence cigarettes/day has a narrower range, resulting in lower statistical strength and misclassification bias. Not recognising the most accurate exposure profile might result in underestimation or overestimation of risk and also biased estimates of effect size. 
Table 2 Generalised linear models comparing adjusted effect size of smoking variables on outcomes

\begin{tabular}{|c|c|c|c|c|c|}
\hline Outcome & Predictor & Standardised $b$ & SE of standardised $b$ & $Z$ value & $P$ value \\
\hline \multirow[t]{3}{*}{$\mathrm{FEV}_{1} / \mathrm{FVC}$} & Duration & -0.310 & 0.012 & & \\
\hline & Cigarettes/day & -0.080 & 0.009 & -15.442 & $<0.001$ \\
\hline & Pack-years & -0.183 & 0.009 & -8.353 & $<0.001$ \\
\hline \multirow[t]{3}{*}{$\mathrm{FEV}_{1}$} & Duration & -0.253 & 0.011 & & \\
\hline & Cigarettes/day & -0.077 & 0.008 & -12.853 & $<0.001$ \\
\hline & Pack-years & -0.165 & 0.009 & -6.32 & $<0.001$ \\
\hline \multirow[t]{3}{*}{ CT emphysema } & Duration & 0.219 & 0.012 & & \\
\hline & Cigarettes/day & 0.058 & 0.009 & 10.385 & $<0.001$ \\
\hline & Pack-years & 0.128 & 0.010 & 5.754 & $<0.001$ \\
\hline \multirow[t]{3}{*}{$\mathrm{CT}$ gas trapping } & Duration & 0.247 & 0.012 & & \\
\hline & Cigarettes/day & 0.077 & 0.009 & 11.009 & $<0.001$ \\
\hline & Pack-years & 0.159 & 0.010 & 5.566 & $<0.001$ \\
\hline \multirow[t]{3}{*}{ 6MWD } & Duration & -0.186 & 0.012 & & \\
\hline & Cigarettes/day & -0.082 & 0.009 & -7.055 & $<0.001$ \\
\hline & Pack-years & -0.150 & 0.009 & -2.387 & 0.008 \\
\hline \multirow[t]{3}{*}{ SGRQ } & Duration & 0.227 & 0.013 & & \\
\hline & Cigarettes/day & 0.148 & 0.010 & 4.907 & $<0.001$ \\
\hline & Pack-years & 0.230 & 0.010 & 0.042 & 0.483 \\
\hline
\end{tabular}

Estimated standardised regression coefficient $b$ is adjusted for age, race, sex, body mass index, scanner type, centre, age of smoking onset and current smoking status. $\mathrm{P}$ value obtained by Z-test for comparing standardised $b$ of smoking duration with that of cigarettes/day and pack-years of smoking.

6MWD, 6 min walk distance; SGRQ, St George's Respiratory Questionnaire.

The duration of smoking is also likely impacted by the age of onset of smoking. Early-onset smoking is likely to impact lung growth and maturation and result in a lower baseline peak lung function which has been shown to be a risk factor for COPD. ${ }^{21}$ We performed a sensitivity analysis with and without adjustment for age of onset and found that adjustment for age of smoking

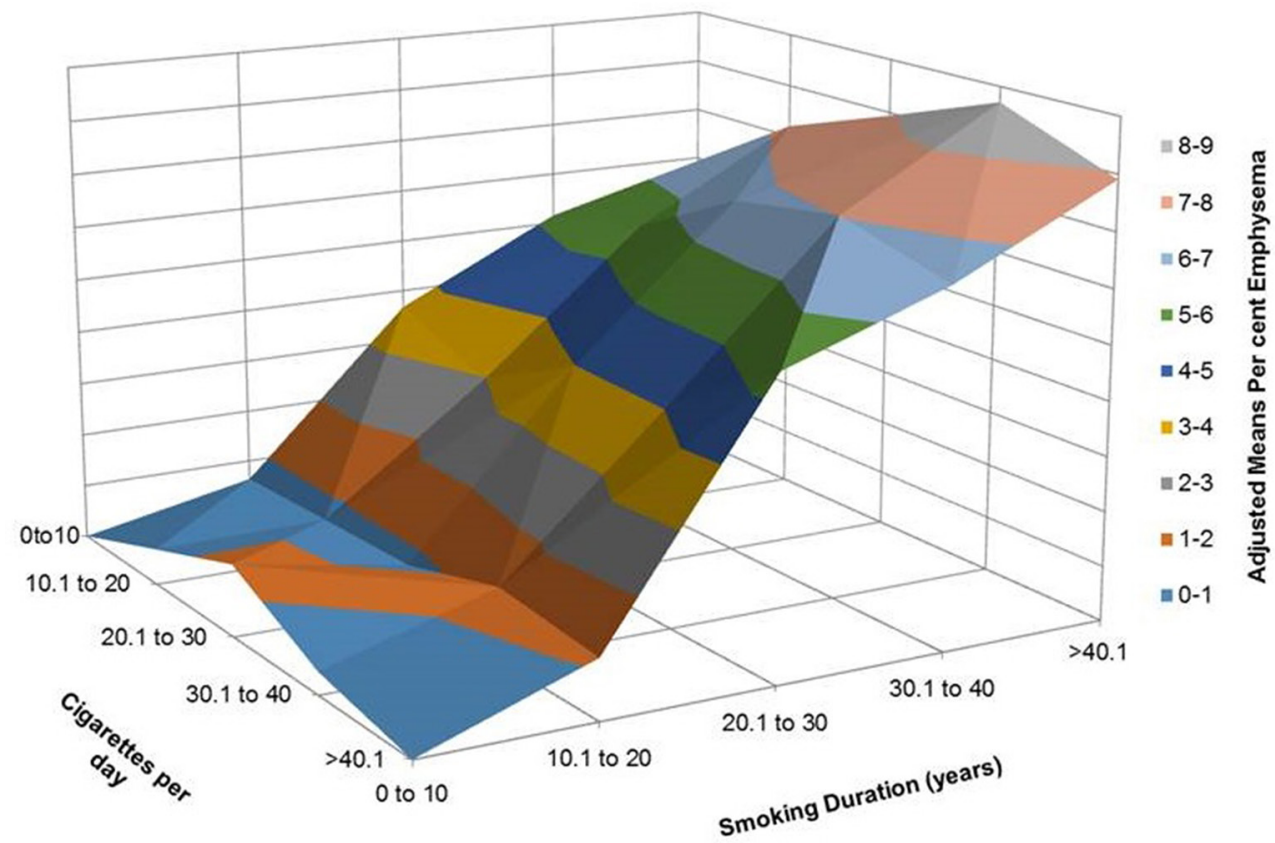

Figure 3 Three-dimensional surface plots demonstrate the relationships between linear slopes for the adjusted means of CT emphysema over categorised cigarettes/day and over categorised smoking duration (years). The adjusted means CT emphysema slopes for cigarettes/day are relatively flat across all duration categories whereas adjusted means CT emphysema increases linearly with increasing smoking duration across all categories of cigarettes/day. All categorisation is based on 10 unit increments. Colour panel on the right shows adjusted means of CT emphysema adjusted for age, race, sex, body mass index, CT scanner type, centre, age of smoking onset and current smoking status. Note that surface plots were drawn for 23 of the 25 combinations of smoking cigarettes/day and smoking duration, and the two combinations with insufficient participants were treated as zero to smoothen the surface plots. 
onset strengthened the relationship between smoking duration and COPD but not that for cigarettes/day and pack-years. Although smoking duration is more likely to be greater with older age, this is also true for the composite of pack-years; we also adjusted for age at enrolment and age of smoking onset.

Our findings have several significant public health implications. First, information on smoking duration alone for estimating risk of COPD is easier to obtain and likely less affected by recall bias and hence is more pragmatic. This is not to say that the number of cigarettes smoked per day does not matter. Although cigarettes/day is likely important and our study was not designed to assess mechanistic relationships, the current methods of measurement with average number of cigarettes/day over time appear to provide inferior risk assessment to that provided by smoking duration. The threshold effect for disease occurrence in COPD may not lie in the cigarettes smoked per day but in the duration of smoking. Our findings reinforce the importance of complete smoking cessation rather than decreasing the number of cigarettes smoked to reduce the risk of lung disease.

Our study has several strengths. This is a large well-characterised cohort of current and former smokers with diversity of race and sex; data collection was blinded to the outcomes, minimising the potential bias in comprehensiveness of exposure assessments which can be impacted by ascertainment of knowledge of disease status. Data on smoking duration and cigarettes/day were meticulously collected by investigators to determine risk, but this level of detail may not be feasible in a busy clinical practice. This is more likely to impact smoking cigarettes/day measurements than duration. The study has some limitations. Participants enrolled in the COPDGene study had to have at least a 10 pack-year smoking history, and the cohort contains a large proportion of participants with COPD with heavy smoking burden and the range of smoking cigarettes/day is likely narrower and more uniform than in a general population sample. Smoking exposure history is subject to recall bias; however, in a disease with a long latency, validated questionnaires are the most accepted method of measuring exposure. We did not include non-smoking controls because using non-smokers as a reference standard tends to overestimate the dose-response relationship as any smoking is likely to be worse than never smoking. Compared with base models, all models with smoking measures contributed incrementally to the COPD outcomes, and this could be due to the inclusion of participants with at least a 10 pack-year smoking history which establishes a baseline smoking exposure. For this reason, we performed additional analyses to study the differential effects of cigarettes/day and duration across the range of each smoking measure. We calculated smoking duration as the difference between the age at smoking onset and the age at smoking cessation, and did not account for possible short periods of smoking cessation; however, our methodology is less subject to recall bias, and is consistent with the methods used in large epidemiological studies. ${ }^{162}$ Findings from the Lung Health Study also showed that the rate of decline of lung function over 11 years was similar between sustained and intermittent smokers, suggesting that periods of intermittent cessation do not significantly impact outcomes. ${ }^{22}$

\section{CONCLUSIONS}

In a large cohort of smokers with and without COPD, smoking duration provides stronger risk estimates of COPD components than cigarettes smoked per day and the composite index of pack-years. Given the limitations of measuring cigarettes/ day, giving equal weightage to smoking cigarettes/day and duration might attenuate the measured strength of association between smoking and COPD, and result in misclassification and biased estimates of disease risk.

\section{Author affiliations}

${ }^{1}$ Division of Pulmonary, Allergy and Critical Care Medicine, University of Alabama at Birmingham, Birmingham, Alabama, USA

${ }^{2} U A B$ Lung Health Center, University of Alabama at Birmingham, Birmingham, Alabama, USA

${ }^{3}$ Division of Preventive Medicine, University of Alabama at Birmingham, Birmingham, Alabama, USA

${ }^{4}$ Department of Biostatistics and Bioinformatics, National Jewish Health, Denver, Colorado, USA

${ }^{5}$ Channing Division of Network Medicine, Brigham and Women's Hospital, Boston, Massachusetts, USA

${ }^{6}$ Division of Pulmonary and Critical Care Medicine, Brigham and Women's Hospital, Boston, Massachusetts, USA

${ }^{7}$ Pulmonary and Critical Care Medicine, Harvard Medical School, Boston,

Massachusetts, USA

${ }^{8}$ Division of Pulmonary, Critical Care and Sleep Medicine, National Jewish Health, Denver, Colorado, USA

${ }^{9}$ Division of Pulmonary and Critical Care Medicine, University of Nebraska Medical Center, Omaha, Nebraska, USA

${ }^{10}$ Clinical Discovery Unit, AstraZeneca, Cambridge, UK

${ }^{11}$ Division of Pulmonary and Critical Care Medicine, Morehouse School of Medicine, Atlanta, Georgia, USA

${ }^{12}$ Division of Pulmonary and Critical Care Medicine, University of California Los Angeles, Los Angeles, California, USA

${ }^{13}$ Division of Pulmonary and Critical Care Medicine, Johns Hopkins University School of Medicine, Baltimore, Maryland, USA

${ }^{14}$ Pulmonary and Critical Care Medicine, Birmingham VA Medical Center, Birmingham, Alabama, USA

Contributors SPB had full access to all of the data in the study and takes responsibility for the integrity of the data and the accuracy of the data analysis. Study concept and design: SPB, YK and WCB. Acquisition, analysis or interpretation of data: all authors. Drafting of the manuscript: SPB. Critical revision of the manuscript for important intellectual content: all authors. Statistical analysis: YK and SPB. Study supervision: all authors.

Funding The project was supported by Awards R01 HL089897 and R01 HL089856 from the National Heart, Lung and Blood Institute. The COPDGene project is also supported by the COPD Foundation through contributions made to an Industry Advisory Board which comprised AstraZeneca, Boehringer Ingelheim, Novartis, Pfizer, Siemens, Sunovion and GlaxoSmithKline. SPB is supported by NIH K23 Grant K23HL133438.

\section{Competing interests None declared.}

Ethics approval The institutional review boards of all 21 participating centres approved the COPDGene study.

Provenance and peer review Not commissioned; externally peer reviewed.

(c) Article author(s) (or their employer(s) unless otherwise stated in the text of the article) 2018. All rights reserved. No commercial use is permitted unless otherwise expressly granted.

\section{REFERENCES}

1 Vestbo J, Hurd SS, Agustí AG, et al. Global strategy for the diagnosis, management, and prevention of chronic obstructive pulmonary disease: GOLD executive summary Am J Respir Crit Care Med 2013;187:347-65.

2 Etter JF, Perneger TV. Measurement of self reported active exposure to cigarette smoke. J Epidemiol Community Health 2001;55:674-80.

3 Jaakkola MS, Jaakkola JJ. Assessment of exposure to environmental tobacco smoke. Eur Respir J 1997;10:2384-97

4 Doll R, Peto R. Cigarette smoking and bronchial carcinoma: dose and time relationships among regular smokers and lifelong non-smokers. J Epidemiol Community Health 1978:32:303-13.

5 Flanders WD, Lally CA, Zhu BP, et al. Lung cancer mortality in relation to age, duration of smoking, and daily cigarette consumption: results from cancer prevention study II. Cancer Res 2003;63:6556-62.

6 Lubin JH, Caporaso NE. Cigarette smoking and lung cancer: modeling total exposure and intensity. Cancer Epidemiol Biomarkers Prev 2006;15:517-23.

7 Regan EA, Hokanson JE, Murphy JR, et al. Genetic epidemiology of COPD (COPDGene) study design. COPD 2010;7:32-43. 
8 Wan ES, Castaldi PJ, Cho MH, et al. Epidemiology, genetics, and subtyping of preserved ratio impaired spirometry (PRISm) in COPDGene. Respir Res 2014;15:89.

9 Madani A, Zanen J, de Maertelaer V, et al. Pulmonary emphysema: objective quantification at multi-detector row CT--comparison with macroscopic and microscopic morphometry. Radiology 2006;238:1036-43.

10 Jones PW, Quirk FH, Baveystock CM, et al. A self-complete measure of health status for chronic airflow limitation. The St. george's respiratory questionnaire. Am Rev Respir Dis 1992:145:1321-7.

11 ATS Committee on Proficiency Standards for Clinical Pulmonary Function Laboratories. ATS statement: guidelines for the six-minute walk test. Am J Respir Crit Care Med 2002;166:111-7.

12 Filion KB, Azoulay L, Platt RW, et al. A multicenter observational study of incretinbased drugs and heart failure. N Engl J Med 2016;374:1145-54.

13 Pandeya N, Williams GM, Sadhegi S, et al. Associations of duration, intensity, and quantity of smoking with adenocarcinoma and squamous cell carcinoma of the esophagus. Am J Epidemiol 2008;168:105-14.

14 Lubin JH, Couper D, Lutsey PL, et al. Risk of cardiovascular disease from cumulative cigarette use and the impact of smoking intensity. Epidemiology 2016;27:395-404.
15 Tashkin DP, Altose MD, Connett JE, et al. Methacholine reactivity predicts changes in lung function over time in smokers with early chronic obstructive pulmonary disease. The lung health study research group. Am J Respir Crit Care Med 1996;153(6 Pt 1):1802-11.

16 Scanlon PD, Connett JE, Waller LA, et al. Smoking cessation and lung function in mild-to-moderate chronic obstructive pulmonary disease. The lung health study. Am J Respir Crit Care Med 2000;161(2 Pt 1):381-90.

17 Nakamura H. Genetics of COPD. Allergol Int 2011;60:253-8.

18 Sze MA, Dimitriu PA, Hayashi $\mathrm{S}$, et al. The lung tissue microbiome in chronic obstructive pulmonary disease. Am J Respir Crit Care Med 2012;185:1073-80.

19 Erb-Downward JR, Thompson DL, Han MK, et al. Analysis of the lung microbiome in the "healthy" smoker and in COPD. PLoS One 2011;6:e16384.

20 Scherer G. Smoking behaviour and compensation: a review of the literature. Psychopharmacology 1999;145:1-20.

21 Lange P, Celli B, Agustí A, et al. Lung-function trajectories leading to chronic obstructive pulmonary disease. N Engl J Med 2015;373:111-22.

22 Anthonisen NR, Connett JE, Murray RP. Smoking and lung function of lung health study participants after 11 years. Am J Respir Crit Care Med 2002;166:675-9. 